\title{
Ultrasound Measurement of Cartilage Thickness in Childhood Arthritis - Target the Tissue, Tailor the Technique
}

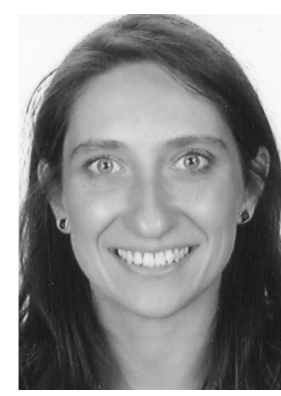

Joint cartilage is a major target of the erosive process in chronic arthritis. The occurrence of cartilage loss in children with juvenile idiopathic arthritis (JIA) represents, therefore, an early indicator of joint damage and raises the need to intensify therapy before irreversible structural changes develop. Until a few years ago, most experience in joint imaging in JIA was based on conventional radiography ${ }^{1}$. Joint space narrowing and erosions seen on plain films have traditionally been considered important markers of disease progression and have been observed early in the disease course in a higher-than-expected proportion of children with $\mathrm{JIA}^{2}$. However, radiographs are inadequately sensitive in the detection of the initial structural changes in JIA.

Moreover, accurate assessment of joint damage in children is complicated by the age-related variations in the thickness of articular cartilage and the ongoing skeletal maturation. In growing children, bones are extensively cartilaginous, and ossification centers appear progressively and complete their growth over several years. In young children the epiphyses are highly vascularized and the metaphyseal vessels anastomose with epiphyseal vessels throughout the growth plate. In children with chronic arthritis, inflammation affecting the epiphyseal cartilage may extend to the ossification centers, causing excessive growth, deformities, or epiphyseal erosions. As a result, irreversible cartilage destruction may occur before bone changes are radiographically evident.

Newer imaging modalities, particularly ultrasound (US) and magnetic resonance imaging (MRI), have played an increasing role in the identification of early signs of synovitis and damage in $\mathrm{JIA}^{3}$. US is particularly suited for use in children because of its noninvasiveness, rapidity of performance, relatively low cost, ability to scan multiple joints at one time, repeatability, safety, and high patient acceptability. US enables visualization of the soft tissues and principal components of the joints and can detect the cartilage of unossified epiphyses and the osseous nuclei before they become visible on plain radiographs. However, US cannot visualize the entire joint because of the limited acoustic window on bones, and is the most operator- and machine-dependent imaging technique ${ }^{4}$. MRI has the unique advantage of allowing the simultaneous assessment of all joint structures and the differentiation of articular from epiphyseal cartilage along the different stages of development ${ }^{5}$. MRI drawbacks include the requirement of sedation in younger children, the capacity to evaluate only 1 or 2 joints in the same session, and the long examination time. In addition, it is expensive and is not uniformly available in all centers.

Growing interest in US and MRI has emphasized the need to improve the knowledge of the imaging anatomy of joints of healthy children, including physiological changes over time, and to establish definitions and standard references specific for the various pediatric ages. Clearly the concepts used in the interpretation of US and MRI images in adults cannot be extrapolated to children with JIA or to healthy children $6,7,8$.

Spannow, et al were the first to attempt the measurement and quantification of cartilage thickness in pediatric subjects. They established the normal ranges of US-detected cartilage thickness in small and large joints by examining a cohort of 394 healthy children. Further, they demonstrated that cartilage was significantly thicker in boys than in girls and that cartilage thickness diminished progressively with age in both sexes ${ }^{9,10}$. Similar variations by sex and age were also found by Panghaal, et al, who used a different approach in the measurement of cartilage thickness in the knees of healthy subjects from birth to 21 years $^{11}$.

In another study, Spannow and coworkers observed good intra- and interobserver agreement in the evaluation of cartilage thickness in several joints, using US standard scans according to European League Against Rheumatism guidelines $^{12}$. They then validated the use of US for measurement of cartilage thickness in the right knee, ankle, wrist, and second metacarpophalangeal and proximal interphalangeal joints in healthy children (9.9-13.3 yrs), by comparing US with MRI findings. Overall, US and MRI

See Cartilage thickness in JIA, page 534 
revealed good agreement, except for the wrist joint ${ }^{13}$. In a subsequent study, the same authors measured cartilage thickness with US in the above target joints on both sides, in 95 patients with JIA (5-15 yrs), and compared the findings with those obtained in healthy children ${ }^{14}$. After controlling for age and sex, cartilage thickness in the knee, wrist, and second interphalangeal joint in patients with JIA was found to be significantly lower than in a healthy cohort. Among patients with JIA, cartilage thickness in the knee was significantly lower in those with polyarthritis and systemic arthritis compared to the oligoarticular group. Notably, the authors observed significantly decreased cartilage thickness in patients with JIA, as compared with the control sample, regardless of whether the examined joints were previously affected. Surprisingly, they found that the group of patients with the shortest disease duration had the lowest cartilage thickness, a finding that was attributed to a higher level of disease activity in those patients.

Published in this issue of The Journal is a report by Pradsgaard, et al from the same group of investigators; their study aimed to validate and compare US versus MRI measurements of distal femoral cartilage thickness of the knee in children with JIA, and to identify the preferential anatomical landmark for cartilage assessment in the distal femur ${ }^{15}$. MRI was selected as the gold standard. However, in spite of the validation studies performed in adult populations ${ }^{16,17}$, MRI has not yet been validated for the measurement of cartilage in children, and therefore neither MRI nor other available imaging techniques can be regarded as the gold standard for such purpose.

Despite the changes in treatment strategies over the years, the knee remains the most commonly affected joint at onset and during followup in children with JIA. Therefore, although US and MRI examinations of joints such as the wrist and ankle, whose involvement has been associated with worse prognosis ${ }^{18}$, may be more helpful to identify patients at higher risk for a poorer outcome, at the present stage, the knee remains the most appropriate joint for outcome evaluation in children with JIA.

Unlike previous studies, in which US measurement of cartilage thickness of the knee was obtained only at the intercondylar notch, in the present analysis Pradsgaard, et al also measure at the medial and lateral condyle. On US, both knees were evaluated according to a very detailed scanning protocol, whereas MRI was performed only in 1 knee, chosen randomly. Twenty-three children with JIA, aged 7.2-15.7 years, were included in the study. Sixteen had persistent oligoarthritis, whereas 7 had extended oligoarthritis. No patient had active arthritis at the time of examination, although active synovitis of the knee was recorded in the medical history of 16 patients.

Cartilage thickness, assessed by US, correlated significantly with MRI measurements in all the 3 sites considered $(r=0.7, p<0.05$ at the intercondylar notch; $r=0.86, p<$
0.001 at the medial condyle; $r=0.71, p<0.001$ at the lateral condyle). Interestingly, the authors noticed that the mean cartilage thickness differed significantly between the 2 imaging modalities in all 3 sites, and found a systematic error in their initial analysis. After correcting the sound velocity within the cartilage tissue, the estimated differences between MRI and US measurements were completely flattened. The results of this study support the validity of US in the assessment of cartilage thickness of the knee in JIA as compared to MRI. Moreover, they highlight the notion that accurate knowledge of the technique and physics of US is fundamental for correct acquisition and interpretation of images, and should never be overlooked.

Cartilage on both US and MRI measurements in knees with a history of active arthritis was thinner than in previously unaffected knees, although the difference was statistically significant only in the lateral condyle. This finding is intriguing if one considers that in the whole cohort US-measured cartilage was significantly thinner in the medial condyle than in the lateral and intercondylar sites, and that MRI showed the same phenomenon, although this was not statistically significant. Their observation suggests that the medial part of the cartilage in the distal femur represents the area where damage appears first, irrespective of whether clinically active arthritis occurred in that knee, whereas the cartilage in the lateral condyle is more affected by the active inflammatory process. Nonetheless, the authors underline that the bony surface at the medial and lateral condyle may be difficult to identify and measure by US during followup, especially in younger children. They conclude that the intercondylar area may be the best site to assess cartilage thickness, because of its easier assessment and lower variability on US as compared to MRI.

Although Pradsgaard, et al are to be commended for addressing such a challenging issue, their results should be further scrutinized in longitudinal studies and in patients with JIA of different categories, joint involvement, disease course, and therapeutic interventions. Further, comparison of US findings with morphologic assessment of the different types of hyaline cartilage and biochemical evaluation of the matrix composition over time through the newer MRI techniques (T2-weighted imaging, delayed contrast-enhanced MR imaging, T2 relaxation mapping ${ }^{19}$ ) is also worthwhile to better understand the pathophysiological changes of cartilage thickness in patients with JIA.

SILVIA MAGNI-MANZONI, MD; IRCCS Ospedale Pediatrico Bambino Gesù, Piazza Sant'Onofrio 4, Rome 00165, Italy.

Address correspondence to Dr. S. Magni-Manzoni.

E-mail: silvia.magnimanzoni@opbg.net

\section{REFERENCES}

1. Babyn A, Doria AS. Radiologic investigations of rheumatic Personal non-commercial use only. The Journal of Rheumatology Copyright @ 2015 . All rights reserved. 
diseases. Pediatr Clin North Am 2005;52:373-411.

2. Ravelli A. The time has come to include assessment of radiographic progression in juvenile idiopathic arthritis clinical trials.

J Rheumatol 2008;35:553-7.

3. Magni-Manzoni S, Malattia C, Lanni S, Ravelli A. Advances and challenges in imaging in juvenile idiopathic arthritis. Nat Rev Rheumatol 2012;8:329-36.

4. Collado P, Jousse-Joulin S, Alcalde M, Naredo E, D'Agostino MA. Is ultrasound a validated imaging tool for the diagnosis and management of synovitis in juvenile idiopathic arthritis? A systematic literature review. Arthritis Care Res 2012;64:1011-9.

5. Ho-Fung VM, Jaramillo D. Cartilage imaging in children. Current indications, magnetic resonance imaging techniques, and imaging findings. Radiol Clin N Am 2013;51:689-702.

6. Magni-Manzoni S, Scirè CA, Ravelli A, Klersy C, Rossi S Muratore V, et al. Ultrasound-detected synovial abnormalities are frequent in clinically inactive juvenile idiopathic arthritis, but do not predict a flare of synovitis. Ann Rheum Dis 2013;72:223-8.

7. Müller LS, Avenarius D, Damasio B, Eldevik OP, Malattia C, Lambot-Juhan $\mathrm{K}$, et al. The paediatric wrist revisited: redefining MR findings in healthy children. Ann Rheum Dis 2011;70:605-10.

8. Ording Muller LS, Boavida P, Avenarius D, Damasio B, Eldevik OP, Malattia $\mathrm{C}$, et al. MRI of the wrist in juvenile idiopathic arthritis: erosions or normal variants? A prospective case-control study. Pediatr Radiol 2013;43:785-95.

9. Spannow AH, Stenboeg E, Pfeiffer-Jensen M, Herlin T. Ultrasound measurement of joint cartilage thickness in large and small joints in healthy children: a clinical pilot study assessing observer variability. Pediatr Rheumatol Online J 2007;5:3.

10. Spannow AH, Pfeiffer-Jensen M, Andersen NT, Herlin T, Stenbøg E. Ultrasonographic measurements of joint cartilage thickness in healthy children: age- and sex-related standard reference values. J Rheumatol 2010;37:2595-601.

11. Panghaal V, Janow G, Trinh A, Ilowite N, Levin TL. Normal epiphyseal cartilage measurements in the knee in children: an alternative sonographic approach. J Ultrasound Med 2012; $31: 49-53$
12. Spannow AH, Pfeiffer-Jensen M, Andersen NT, Stenbøg E, Herlin T. Inter- and intraobserver variation of ultrasonographic cartilage thickness assessments in small and large joints in healthy children. Pediatr Rheumatol Online J 2009;7:12.

13. Spannow AH, Stenboeg E, Pfeiffer-Jensen M, Fiirgaard B, Haislund $\mathrm{M}$, Ostergaard M, et al. Ultrasound and MRI measurements of joint cartilage in healthy children: a validation study. Ultraschall Med 2011;32 Suppl 1:S110-6.

14. Pradsgaard D $\varnothing$, Spannow AH, Heuck C, Herlin T. Decreased cartilage thickness in juvenile idiopathic arthritis assessed by ultrasonography. J Rheumatol 2013;40:1596-603.

15. Pradsgaard D $\varnothing$, Fiirgaard B, Spannow AH, Heuck C, Herlin T. Cartilage thickness of the knee joint in juvenile idiopathic arthritis: comparative assessment by ultrasonography and magnetic resonance imaging. J Rheumatol 2015;42:534-40.

16. Døhn UM, Conaghan PG, Eshed I, Boonen A, Boyesen P, Peterfy $\mathrm{CG}$, et al. The OMERACT-RAMRIS rheumatoid arthritis magnetic resonance imaging joint space narrowing score: intrareader and interreader reliability and agreement with computed tomography and conventional radiography. J Rheumatol 2014;41:392-7.

17. Fujinaga Y1, Yoshioka H, Sakai T, Sakai Y, Souza F, Lang P. Quantitative measurement of femoral condyle cartilage in the knee by MRI: validation study by multireaders. J Magn Reson Imaging 2014;39:972-7.

18. Beukelman T, Patkar NM, Saag KG, Tolleson-Rinehart S, Cron RQ, DeWitt EM, et al. 2011 American College of Rheumatology recommendations for the treatment of juvenile idiopathic arthritis: initiation and safety monitoring of therapeutic agents for the treatment of arthritis and systemic features. Arthritis Care Res 2011;63:465-82.

19. Restrepo R, Lee EY, Babyn PS. Juvenile idiopathic arthritis. Current practical imaging assessment with emphasis on magnetic resonance imaging. Radiol Clin N Am 2013;51:703-19.

J Rheumatol 2015;42:360-2; doi:10.3899/jrheum.141558 